\title{
The luminosity-specific Planetary Nebulae density in Local Group galaxies
}

\author{
R.L.M. Corradi ${ }^{1,2}$, A. Buzzoni ${ }^{3}$, \& M. Arnaboldi ${ }^{4,5}$ \\ ${ }^{1}$ Isaac Newton Group of Telescopes, Ap. de Correos 321, 38700 Sta. Cruz de la Palma, Spain \\ email: rcorradi@ing.iac.es \\ ${ }^{2}$ Instituto de Astrofísica de Canarias, 38200 La Laguna, Tenerife, Spain \\ ${ }^{3}$ INAF Osservatorio Astronomico di Bologna, Via Ranzani 1, 40127 Bologna, Italy \\ email: alberto.buzzoni@bo.astro.it \\ ${ }^{4}$ ESO Karl-Schwarzschild-Str. 2, 85748 Garching bei München, Germany \\ email: marnabol@eso.org \\ ${ }^{5}$ INAF Osservatorio Astronomico di Torino, Via Osservatorio 20, 10025 Pino Torinese (To), \\ Italy
}

\begin{abstract}
The value of the $\alpha$ ratio, the number of PNe per unit bolometric luminosity in a galaxy, is computed using stellar population synthesis models covering the whole range of Hubble types of galaxies.

Model predictions are compared with the PNe counts in the Local Group, which indicate a fairly constant value of $\alpha$-between 1 and 6 PNe per $10^{7}$ solar luminosities - along the Hubble sequence.
\end{abstract}

Keywords. planetary nebulae; general. galaxies: stellar content

We address the general problem of using planetary nebulae $(\mathrm{PNe})$ as tracers of stellar populations in galaxies and other stellar systems. The PN number per unit galaxy (bolometric) luminosity, known as the " $\alpha$ ratio" given by $\alpha=\mathrm{N}_{P N} / \mathrm{L}_{\text {gal }}$, is computed using population synthesis models applied to simple stellar populations (SSPs) as well as to elaborated galaxy templates covering the full range of star-formation histories as displayed by the different Hubble morphological types. According to the SSP theory, $\alpha$ is set by two parameters, the so-called "specific evolutionary flux", which is tightly constrained by stellar evolution theory, and the $\mathrm{PN}$ visibility lifetime $\mathrm{t}_{P N}$, a much more uncertain quantity. We have estimated $t_{P N}$ as a function of the Post-AGB (PAGB) stellar mass taking into account the leading physical mechanisms at work, that is:

- the PAGB energetic budget,

- dynamical timescale for nebula evaporation,

- transition time between AGB completion and nebula "firing up",

- minimum core mass for stars to experience the thermally pulsing AGB phase,

- initial-to-final mass relation (IFMR; various relationships have been considered).

Fig. 1 shows the predicted dependence of $\alpha$ along the Hubble sequence, compared with the numbers obtained by the updated (and deepest) PN census in Local Group galaxies (Corradi \& Magrini 2006). For each galaxy, the PN number observed within the completeness limit of each survey has been extrapolated 8 mag down the bright cutoff of the PN luminosity function, in order to size up the whole PN galaxy population and derive the empirical value of $\alpha$, which is indicated, with its error bars.

The remarkable feature of the plot is the fairly constant value of $\alpha$-between 1 and 6 PNe per $10^{7}$ solar luminosities - along the Hubble sequence. This implies that: 


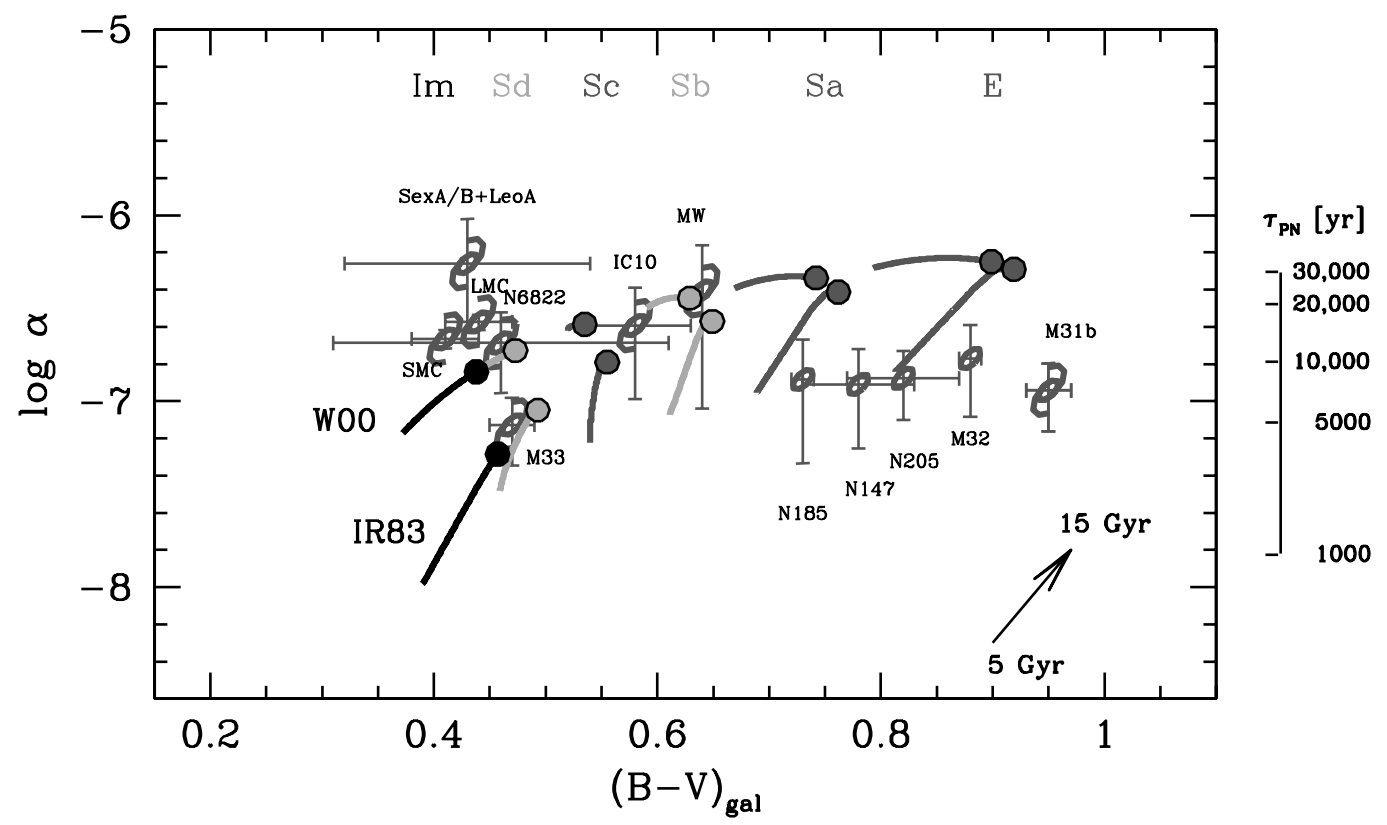

Figure 1. A comprehensive summary of the luminosity-specific PN number in Local Group galaxies. PN data are from the "Local Group Census Project" of Corradi \& Magrini (2006). Also superposed on the plot, there are the Buzzoni $(2002,2005)$ template galaxy models. Theoretical galaxy evolution is tracked along the whole E-Sa-Sb-Sc-Sd-Im Hubble morphological sequence from 5 to $15 \mathrm{Gyr}$, as labeled on the plot. Two model sequences are reported assuming an IFMR as from the standard case of a Reimers (1975) mass loss parameter $\eta=0.3$ according to the Iben \& Renzini (1983) calibration (lower sequence, IR83), and from the empirical relation of Weidemann (2000) models (upper sequence, W00). An indicative estimate of the mean representative PN lifetime (in years) is sketched on the right scale, according to our stellar population synthesis models.

- data favour the empirical IFMR by Weidemann (2000), corresponding to a stronger mass loss for the PN progenitors compared to the standard scenario for Pop II stars as in globular clusters;

- the PN population is dominated by a narrow range of PAGB core masses $(<0.60-0.65$ $\left.\mathrm{M}_{\odot}\right)$;

- the lifetime of a prevailing fraction of PNe is constrained by the dynamical timescale of nebula evaporation rather than the stellar core mass evolution, supporting a small dependence of the $\alpha$ ratio with age and distance, and hence a "universality" of the PN luminosity function.

Full details of these calculations and results can be found Buzzoni et al. (2006).

\section{References}

Buzzoni, A. 2002, AJ 123, 1188

Buzzoni, A. 2005, MNRAS, 361, 725

Buzzoni, A., Arnaboldi, M., \& Corradi, R.L.M. 2006, MNRAS 368, 877

Corradi, R.L.M., \& Magrini, L. 2006, in "Planetary nebulae beyond the Milky Way", J. Walsh,

L. Stanghellini \& N. Douglas eds., Springer, p. 36

Iben, I.Jr., \& Renzini, A. 1983, ARA\&A 21, 271

Reimers, D. 1975, Mem. Soc. Roy. Sci. Liège, 6th Ser., 8, 87

Weidemann, W. 2000, A\&A 363, 647 